\title{
Reflexiones en torno a la responsabilidad social de las empresas empresarial \\ DOI: 10.22403/UQROOMX/TYP03/10
}

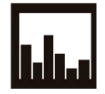

RESUMEN

Jorge Antonio Valderrama, María Guadalupe Velázquez, Juan Carlos Flores Trejo, Oscar Ramón Sánchez López*

La responsabilidad social empresarial conforma una aportación positiva, manifiesta en la planeación, ejecución y control de una práctica cotidiana en favor de la empresa y de la sociedad; considera la minimización de los impactos desfavorables sobre la utilización de recursos, las personas involucradas y el entorno natural y social inmediato y extendido. Además de reflexionar y aprender de las experiencias de éxito empresarial por prácticas socialmente responsables, una manera de iniciar el proceso de responsabilidad empresarial para contextos nacionales, regionales y locales es a través de la aplicación de tablas de sustentabilidad, las cuales permiten conocer el pensamiento de los empresarios sobre su propia responsabilidad en el desarrollo de sus puestos y la forma y alcance de sus acciones, programas y procedimientos de apoyo a la eficiencia, valoración y atención de su gestión económica, social y ambiental.

Palabras Competitividad, empresas eficientes, responsabilidad social,

CLAVE sustentabilidad, tablas comparativas de sustentabilidad.

*Universidad Autónoma de Baja California 


\section{Introducción}

Hoy más que nunca existe la urgente necesidad de operar empresas eficientes. La responsabilidad de ahorrar recursos y de utilizarlos racionalmente está vinculada a la producción y oferta de bienes y servicios efectivos, acordes a las expectativas de los complejos mercados y de una sociedad cada vez más racional, demandante de calidad en sentido amplio.

Esta situación ha provocado la necesidad de cambiar el paradigma decisional tradicional, de un enfoque de optimización de costos por uno de enfoque multicriterio que supere intereses exclusivamente particulares y de visión limitada,y abra posibilidades reales de bienestar socialmente extendidos, conscientemente provocados.

Se reconoce cada vez más la influencia del entorno en la gestión, toda vez que las empresas no pueden trabajar en forma aislada, ya que forman parte de la comunidad que las acoge. Es necesario que la empresa trabaje por sus objetivos, su permanencia y desarrollo, considerando sus propios recursos y sus responsabilidades ampliadas para el mejoramiento de sí misma y de la sociedad, que apoye una sana ecología y una mejor calidad de vida para todos.

\section{Componentes de la responsabilidad empresarial}

Aspectos centrales de la responsabilidad social empresarial son la aplicación voluntaria, decidida y razonada de su implementación, así como su carácter benéfico y extensivo, que cubra la perspectiva de los propietarios y gerentes, de su personal, de sus proveedores y de la sociedad en su conjunto.

La responsabilidad social empresarial conforma una aportación positiva, manifiesta en la planeación, ejecución y control de una práctica cotidiana por parte de las unidades productivas en favor de sí mismas y de la sociedad, que considere minimizar los impactos desfavorables en la gestión sobre la utilización de recursos, a las personas involucradas y el entorno natural inmediato y extendido; estrategia valiosa que asocia las variables de competitividad con sustentabilidad.

De acuerdo con Gitman y McDaniels, la competitividad, vista como la base económica sobre la cual se financia la práctica sustentable, muestra que la empresa: 
al mismo tiempo que busca obtener utilidades (responsabilidad económica), también obedece las leyes (responsabilidad jurídica), haciendo lo correcto, justo y equitativo (responsabilidad ética) siendo un buen ciudadano corporativo (responsabilidad filantrópica). Estos cuatro componentes son distintos, pero juntos forman un todo. No obstante, si la empresa no obtiene utilidades, las otras tres responsabilidades carecen de sustento [Gitman y McDaniels, 200I: I07].

De esta manera se reconoce que la empresa debe ser rentable para poder sobrevivir, su responsabilidad económica se encuentra en la base de la pirámide. En el segundo nivel de la estructura se establece que las consideraciones de orden jurídico también son necesarias para el bienestar empresarial. Por su parte, las responsabilidades éticas descansan sobre la base de las económicas y de las jurídicas. Las responsabilidades filantrópicas se encuentran en la parte más alta del triángulo y sólo se pueden incorporar cuando las responsabilidades anteriores han sido atendidas.

Actualmente, algunas empresas consideran que ser socialmente responsable representa la forma y el costo de hacer negocios; que se requiere invertir en prácticas laborales globales, cuestiones ambientales, prácticas empresariales sostenibles, cuestiones gubernamentales y gestiones públicas (incluidas filantropía y el trabajo voluntario en favor de la comunidad).

Esta condición mejora las utilidades de las empresas, ya que los clientes toman decisiones mejor informadas acerca de dónde comprar y a quién comprar; generalmente, estas decisiones se basan en qué representa una compañía y cuáles son sus valores y aportaciones a la comunidad, definiendo en la práctica responsable la buena ciudadanía corporativa [Ferrel y Hirt, 2004:48].

La responsabilidad social empresarial alienta una efectiva y cotidiana operación administrativa al ampliar los escenarios de actuación e incorporar actores responsables y conscientes de la actividad productiva, que además de aportar recursos, los optimicen, generen mayores beneficios y al mismo tiempo minimicen el impacto negativo sobre las personas y el entorno inmediato extendido. De esta manera, asocia objetivos económicos, sociales y medioambientales.

Las empresas no pueden trabajar en forma aislada, porque forman parte de la comunidad, y ésta es afectada por las actitudes empresariales. Es necesario que la empresa trabaje en el mejoramiento de la sociedad y propicie un am- 
Reflexiones en torno a la

responsabilidad social

biente interno en el que los empleados desarrollen una filosofía que lleve a una sana ecología y una mejor calidad de vida para todos. "Cada empresario tiene que estar convencido de que el futuro de su empresa, su familia y la comunidad depende de sus actitudes y acciones pro ecológicas” (Kras, 1994:75).

\section{Responsabilidad y rentabilidad}

Un nuevo estilo de administración es aquel que procura la incorporación de personal en proceso continuo de avance, sensible y pro activo que, interiorizado en la actividad diaria, desarrolle habilidades, valores y aptitudes; lo cual aporta conocimiento y estrategias para una amplia participación en busca de eficiencia y productividad, a través de prácticas operativas de ahorro de energía, gestión adecuada de recursos, reutilización de residuos y subproductos, entre otras.

Este tipo de operación empresarial estimula un ambiente interno favorable, donde la valoración del recurso humano es una constante, lo que se refleja en un trabajo que genera procesos analíticos participativos, de comunicación fluida, de opciones y de visión ampliada. Las empresas sustentables reconocen oportunidades, son innovadoras y desarrollan una amplia sensibilidad al entorno interno y externo; reconocen la necesidad de extender beneficios al ámbito próximo y cercano del que forman parte, saben que invertir para reducir los impactos negativos de su gestión mejora condiciones de vida de la población en su conjunto; acrecientan su participación en programas y actividades en favor de los empleados, de sus familias, de sectores sociales necesitados y en el bienestar general de la comunidad.

Se identifican más implicaciones de mercado a partir de valores sociales y medioambientales desarrollados por las empresas. Los precios y la calidad de los bienes, la higiene, la seguridad y, en general, el manejo de los procesos, se relaciona cada vez más con los principios éticos de los mensajes publicitarios, sus componentes y presentaciones, de los usos y desusos y de los servicios posventa; esto fortalece la imagen corporativa y el posicionamiento de productos y marcas.

Las asociaciones, fusiones comerciales y los más importantes proveedores de insumos y materias primas son evaluados, al igual que la empresa misma, por los consumidores. Esta consideración forma parte del esquema moderno de clientes cada vez más informados, exigentes e interconectados con una serie de 
productos y servicios globalmente identificados y sobre los que se sustentan amplias expectativas tecnológicas, comerciales, ambientales y sociales. Bajo esta condición mercadológica, las empresas responsables son identificadas y valoradas socialmente, lo que potencia su rentabilidad.

\section{Responsabilidad y sustentabilidad}

La responsabilidad empresarial se encuentra manifiesta en una serie de decisiones conscientes y razonadas:

no porque se lo obliga una ley o simplemente porque está de moda, el concepto está más allá de la legislación; intenta llegar a donde la legislación no llega, ya que lo primero que debe tener en cuenta una empresa socialmente responsable es que debe cumplir con todas las normas jurídicas vigentes, tanto nacionales como internacionales [Rensi, 2004:2]

sin importar tamaño o condición jurídica y si son empresas nacionales o extranjeras.

Considerando lo anterior, se perfilan empresas abiertas a incorporar factores de sustentabilidad ambiental en su gestión operativa, pero son un sector lamentablemente pequeño que se presenta cerrado y lejano para ingresar a sistemas de regulación y aplicación de instrumentos más exigentes y solidarios con el ambiente.

Algunas empresas aplican recursos de reingeniería en procesos críticos, lo que ayuda a reducir emisiones de contaminantes a la atmósfera e impacta responsablemente al disminuir la contaminación directa; de igual forma, estimulan la recuperación de desechos que van desde la inducción hasta la recuperación de efectos negativos; todos relacionados con producción limpia y manejo ecoeficiente por la incorporación y uso de tecnología y manufactura alternativa. Asimismo, dan mantenimiento adecuado a equipo y maquinaria, implementan programas de ahorro y uso responsable de energía, de aprovechamiento racional y reducción de costos, entre otras acciones. La inversión realizada en este sentido reclama el manejo estratégico del presupuesto y su recuperación, como apoyo a una acertada toma de decisiones.

La seguridad y el manejo limpio a lo largo del proceso, y su inclusión como valor intrínseco en la marca y en los productos, se asocian al reconocimiento 
interno y externo a través de la identificación positiva de la conducta y praxis empresarial en favor del medioambiente; muestra una mayor complementariedad entre el desarrollo de la actividad empresarial y la protección del patrimonio natural, relacionando la rentabilidad privada que genera recursos económicos amplios y superiores en relación con la inversión realizada, que bajo el encuadre global de responsabilidad social incide positivamente en la preservación y en una mejor calidad de vida para todos.

\section{Algunas experiencias para reflexionar}

El trabajo Accountability \& Business for Social Responsability, presentado por Campbell (2004) en el Seminario Internacional de Competitividad y Responsabilidad Social muestra evidencia empírica de cómo el enfoque de los negocios, desde la óptica de responsabilidad corporativa, inspira una práctica orientada al desarrollo económico, la cual registra amplios estándares emergentes de significación social y ambiental que tienden a la sustentabilidad, favoreciendo su comprensión.

Se encontró que las elecciones económicas que la empresa realiza llevan a resultados sociales y ambientales manifiestos, tanto en lo positivo como en lo negativo; por lo anterior, las empresas necesitan ser administradas de una manera socialmente responsable. Las empresas líderes están conscientes de que el escenario económico no sólo implica relaciones directas y exclusivas con inversionistas, ya que éstas impactan en diferentes contextos, y desarrollan posibilidades de responder a preocupaciones particulares de la comunidad.

El estudio realizado recomienda implementar un sistema de medición y presentación de resultados de impacto económico, relacionado con la toma de decisiones y manejo de riesgos, y presenta un modelo de revisión continua para el manejo de desarrollo económico con las comunidades. Además, la investigación sugiere una serie de cuestionamientos para las empresas e indica la necesidad de apegarse al respeto de todos los aspectos que mantienen a una comunidad sustentable. Concluye que el éxito económico de las empresas depende del modelo de negocio definido e implementado. El reporte final fue distribuido globalmente a través de las redes de socios de las empresas participantes en el estudio $y$, a través de este foro, a la comunidad interesada en la temática. 
Por otra parte, han surgido una serie de cuestionamientos como los plasmados en Beyond Reporting: Creating Business Value And Accountability del World Business Council for Sustainable Development (Engen y Di Piazza, 2005), en el que se señala que se debe pensar en responsabilidad social ahora porque existe una crisis de confianza hacia los negocios, la que se pone de manifiesto en este estudio. En una encuesta aplicada por Globescan a más de 2I 000 personas en junio del 2003, se encontró que las ONG fueron otra vez reconocidas como las más confiables, y las compañías globales resultaron ser las que inspiraron menos confianza.

Una serie de incidentes han provocado protestas extendidas del público y los inversionistas y han impulsado a los legisladores a revisar leyes y ordenanzas relacionadas con dirección corporativa en los Estados Unidos. Aún con estas nuevas salvaguardas persiste la duda en la actuación correcta de los negocios, pero además se descubre que, cómo ilustra este reporte, un creciente número de compañías visionarias han empezado a usar esta presión para conducir sus negocios hacia una dirección diferente, construyendo una nueva confianza y desarrollando nuevas oportunidades comerciales como resultado [Engen \& Di Piazza, 2005:4].

Ante tal circunstancia, algunos líderes empresariales han reaccionado con visión y prontitud, y desarrollado condiciones de respuesta efectiva. Como evidencia sumada el estudio referido, se establece que la práctica empresarial socialmente responsable es la estrategia correcta. Empresas de investigación, tales como Innovest, opinan que las compañías que han comprendido este hecho y actúan conforme a su responsabilidad con la sociedad han logrado mejores resultados financieros por prontos retornos de inversión. Igualmente, la investigación empírica de Deutsche Bank London ha sugerido un vínculo fuerte y positivo entre dirección corporativa y de actuación de dividendos.

\section{Aplicación y medición de la responsabilidad}

Para contextos nacionales, regionales y locales:

Una manera de medir los niveles de responsabilidad empresarial es a partir del conocimiento de lo que los empresarios piensan sobre su propia responsabilidad en el desarrollo de su puesto y como actúan, ya que la responsabilidad con el 
Reflexiones en torno a la

responsabilidad social

medioambiente es fundamentalmente una decisión económica-administrativa, así como el tipo y el alcance puede ser un área para evaluar el compromiso que se tiene, las acciones, los programas y procedimientos implantados será lo que revele la seriedad de la intención de apoyar la eficiencia, el recurso humano integrado y el medioambiente [Velázquez-Valderrama, 2003: 146].

Las tablas comparativas de sustentabilidad y no sustentabilidad conforman un instrumento operativo que permite medir las actividades que en torno a la responsabilidad social están implementando las empresas, se trata de un acercamiento inicial que genera resultados en dos ámbitos: uno empresarial y otro global; esto favorece la identificación de una actuación objetiva.

Los ámbitos de actuación empresarial van, desde la forma de pensar, que deberá responder a una sana administración, planeación, organización, dirección y control; hasta la preparación suficiente y oportuna para atender la responsabilidad, pasando por la comunicación, la producción e impacto social, para llegar a los aspectos físicos del manejo de los problemas ambientales; asimismo, a nivel global, atiende aspectos donde la cultura, la tecnología, el crecimiento económico y el nivel de vida se asocian a variables medibles para identificar niveles de sustentabilidad y no sustentabilidad.

El más significativo aporte de este instrumento de trabajo atiende a los aspectos metodológicos definidos, que con respuestas claras y precisas, puede conducir a la implementación de formas de trabajo validadas por las empresas a fin de ajustar sus procedimientos administrativos cotidianos para que operen bajo estructuras sencillas de bajos costos y amplios beneficios sociales [VelázquezValderrama, 2003: 160].

Toda experiencia empresarial que lleve al conocimiento e identificación de resultados mediales de sistemas de gestión ambiental debería ser evaluada y proyectada socialmente, ya que el problema de contaminación y manejo de desechos, entre otros, son una exigencia creciente para las empresas de todo tipo, tamaño y estructura. Bajo esta lógica, la instrumentación de acciones y programas de manera dispersa y aislada no permite el avance y la operación de medidas y mejoras, así como la valoración y establecimiento de estrategias preventivas. La falta de registros e involucramientos al interior y al exterior puede provocar no sólo desorden e ineficiencia, sino también, costos privados y sociales linealmente crecientes. El desconocimiento de experiencias em- 
presariales por ramo, especialidad o contexto redundan en apatía, negación y pérdida de conciencia.

\section{Reflexiones finales}

Fortalecidos en los anteriores razonamientos, en el corto y largo plazo los retos empresariales serán cada vez mayores en términos de eficiencia, competitividad, rentabilidad y sustentabilidad, vislumbrando en perspectiva razonada y aprendida la demanda de una mayor responsabilidad social empresarial, como una posibilidad de bienestar privado socialmente extendido.

Las empresas no pueden trabajar en forma aislada ya que forman parte de la comunidad que las acoge, y ésta es afectada por sus acciones y decisiones, es necesario que la empresa trabaje por sus objetivos, su permanencia y desarrollo, considerando sus propios recursos y sus responsabilidades ampliadas para el mejoramiento de sí misma y de la sociedad, apoyando una sana ecología y una mejor calidad de vida para todos.

La responsabilidad social empresarial conforma una aportación positiva, manifiesta en la planeación, ejecución y control de una práctica cotidiana por parte de las unidades productivas en favor de sí mismas y de la sociedad, que considere la minimización de los impactos desfavorables en la gestión sobre la utilización de recursos, las personas involucradas y el entorno natural inmediato y extendido.

Para contextos nacionales, regionales y locales, una manera de medir los niveles de responsabilidad empresarial es conociendo lo que piensan los empresarios sobre su propia responsabilidad en el desarrollo de su puesto y cómo actúan, ya que la responsabilidad con el medio ambiente es fundamentalmente una decisión económica-administrativa, así como el tipo y el alcance, pueden ser áreas para evaluar el compromiso que se tiene, las acciones, los programas y procedimientos implantados serán los que revelen la seriedad de la intención de apoyar la eficiencia, el recurso humano integrado y el medio ambiente.

Las tablas comparativas de sustentabilidad y no sustentabilidad conforman un instrumento operativo para medir las actividades que en torno a la responsabilidad social están implementando las empresas; se trata de un acercamiento inicial que genera resultados en dos ámbitos: uno empresarial y otro global, favoreciendo la identificación de una actuación objetiva. 
El más significativo aporte de la aplicación de tablas comparativas de sustentabilidad y no sustentabilidad atiende a los aspectos metodológicos definidos, que con respuestas claras y precisas pueden conducir a la implementación de formas de trabajo validadas por las empresas a fin de ajustar sus procedimientos administrativos cotidianos para que operen bajo estructuras sencillas de bajos costos y amplios beneficios sociales.

\section{FueNTES CONSULTADAS}

Campbell, Hellen (2004). “Accountability \& Business for Social Responsibility”. Ponencia presentada en el Seminario Internacional Competitividad y Responsabilidad Social. Santiago de Chile, junio de 2004.

Engen, Travis y Di Piazza, Samuel (2005). Beyond Reporting: Creating Business Value And Accountability delWorld Business Council for Sustainable Development.

Ferrel, O.C. Hirt, Geoffrey A. Adriaenérs, M. Flores, M.A. y Ramos, L. (2004). Introducción a los negocios en un mundo cambiante. México: McGraw-Hill Interamericana.

Gitman, Larry J. y McDaniels Carl (200I). El futuro de los negocios, México: International Thomson.

Kras, Eva (1994). El desarrollo sustentable y las empresas. México: Editorial Iberoamericana.

Rensi, Roberto (2004)."La visión de Unión Europea sobre competitividad y responsabilidad social empresarial”. Ponencia presentada en el Seminario Internacional Competitividad y Responsabilidad Social. Santiago de Chile, 2004.

Velázquez R., María Guadalupe y Valderrama M., Jorge A. (2003). “Aplicación de criterios de sustentabilidad en empresas turísticas en la frontera norte de México. Estudio exploratorio con propuesta de atención y seguimiento", en Turismo I. Organizaciones del tercer sector en el turismo. México, 2003. 